\title{
Microglia and monocytes: molecularly defined
}

\author{
Marco Prinz
}

Published online: 8 August 2014

(C) Springer-Verlag Berlin Heidelberg 2014

This issue of Acta Neuropathologica includes a cluster of four review articles on microglia, macrophages and monocytes and their roles in CNS pathologies, which appears more than 150 years after the first description of glia by the German pathologist Rudolf Virchow in 1858. He was just 37 years old when he gave a series of 20 lectures for colleagues and medical practitioners in Berlin. In the 13th lecture, he described "connective tissue originating from the brain and protruding onto the brain's surface" and this was actually the birth of the term "glia". It took more than six decades to the description of microglia when Pio del Rio Hortega discovered microglia as the "third element" different from macroglia and neurons by applying improved silver staining methods formerly established by his teacher and Nobel Prize winner Ramon Cajal.

A new era of microglia research has just begun. The renaissance of both basic and clinically linked research on myeloid cells is based on recently developed new methods in genetics and imaging that help to redefine the role of microglia for brain development and disease. Given the importance of microglia in physiological brain functions, it is not surprising that an increasing number of microgliarelated genes have now been associated with neuropsychiatric and neurologic disorders. It is therefore tempting to speculate that we will soon discover a new class of "microgliopathies" in which microglia dysfunction is the primary disease-causing mechanism.

\footnotetext{
M. Prinz $(\square)$

Institute of Neuropathology, University of Freiburg,

Breisacher Str. 64, 79106 Freiburg, Germany

e-mail: marco.prinz@uniklinik-freiburg.de

M. Prinz

BIOSS Centre for Biological Signalling Studies,

University of Freiburg, Freiburg, Germany
}

This said, the present cluster thus represents an opportunity to take stock of a remarkable epoch in microglial research.

One article in this issue acknowledges the origin of microglia and other myeloid cells in the brain and their position in the universe of myeloid cells within the body. Despite the fact that many tissue macrophages in the body already develop prior to birth, namely during early embryogenesis where they are derived from the yolk sac, they all have their own tissue-specific functions. Jung, Prinz and colleagues discuss microglia function during brain development and disease and highlight their interaction with their circulating and sessile relatives.

Kipnis, Meyer-Luehmann and colleagues discuss new technologies in animal models for studying microglia. Especially the application of two-photon imaging for microglia in the living experimental specimen shed new light on microglia behavior during health and disease. This is why microglia are nowadays considered as highly mobile and never resting cells within the CNS. The authors bring the concepts of microglia-mediated neuromodulation and maintenance of homeostasis to the bedside in their account of microglia in neuropsychiatric and developmental disorders, a topic which received widespread attention with proof-of-principle research conducted in a Rett syndrome mouse model.

Glass and Synowitz dive into the diversity of brain tumor-associated macrophages/monocytes that can be derived from either the pool of circulating cells or from brain endogenous sources. The authors discuss animal models for studying myeloid function, emphasizing that the field made major strides by studying brain tumors molecularly. Further clinical progress in the treatment of brain tumors will certainly depend on our in depth understanding of myeloid cell function in this disease entity. 
Cartier and Rossi finally delineate developmental origins of yolk sac-derived microglia as compared to bone marrow-derived monocytes and speculate on how this knowledge can help further therapeutic attempts to engineer hematopoietic cells to engraft the disordered brain for patient benefit. This bench-to-bedside research shows how myeloid cells can be used to improve both experimental and clinical neurodegeneration such as in leukodystrophies.
Together, these reviews encapsulate our present understanding of microglia, and provide an objective-correlative vision of contemporary research methods and concepts. Given this progress, it is more than thrilling to anticipate exciting research papers on microglia and the next microglia cluster to be published in Acta Neuropathologica. 